\title{
Zebrafish Embryo as an Emerging Model Organism in Neurodevelopmental Toxicity Research
}

\author{
Sukriye Caliskan ${ }^{1}$ (D), Ebru Emekli-Alturfan² \\ 'Marmara University, Institute of Health Sciences, Department of Biochemistry, Istanbul, Turkey \\ ${ }^{2}$ Marmara University, Faculty of Dentistry, Department of Basic Medical Sciences, Istanbul, Turkey
}

ORCID IDs of the authors: S.C. 0000-0002-7576-4967; E.E.A. 0000-0003-2419-8587

Please cite this article as: Caliskan S, Emekli-Alturfan E. Zebrafish Embryo as an Emerging Model Organism in Neurodevelopmental Toxicity Research. Eur J Biol 2021; 80(2): 179-187. DOI: 10.26650/EurJBiol.2021.1006402

\begin{abstract}
Zebrafish is a model organism that has become increasingly popular in recent years due to some of the advantages it has when compared to traditional model organisms. Its genetic similarity with humans has contributed significantly to the elucidation of the molecular mechanisms underlying diseases. Moreover, external fertilization and rapid embryonic development of zebrafish embryos have made it attractive in many research areas. The genome of humans and zebrafish are found to be highly conserved having $76-82 \%$ of the disease genes in humans that are also present in zebrafish. Zebrafish have been used in different studies in several concepts of neurogenesis. Unlike mammals, the external development of a zebrafish embryo makes it accessible for experimental manipulation in central nervous system research. It was observed that neurotoxic agents induced similar responses to other vertebral models in zebrafish embryos, whose brain development and blood-brain barrier were similar to those of other vertebrates. This review provides brief information about the availability of zebrafish embryos in neurodevelopmental toxicity research while giving brief information on embryogenesis and neurogenesis in zebrafish. Evaluation of neurotoxicity and the specific effects of various neurotoxins on motor and dopaminergic neurons, neuronal proliferation, mobility, and neurodevelopment are also explained.
\end{abstract}

Keywords: Zebrafish, zebrafish embryo, neurotoxicity, neurodevelopment

\section{INTRODUCTION}

The zebrafish (Danio rerio) is gaining popularity as a model organism in many human diseases as a small tropical freshwater fish. Since the zebrafish shares a high genetic similarity to humans, it is a powerful model for both identifying the actual mechanisms and improving therapeutic strategies in many diseases, including metabolic and neurological diseases (1-3). The genome of humans and zebrafish are found to be highly conserved having $76-82 \%$ of the disease genes in humans that are also present in zebrafish. On the other hand, nearly 20$24 \%$ of genes in zebrafish are duplicated (4). A high genetic similarity to humans enables discoveries of many genetic mutations leading to different hereditary neurological diseases in humans (5). To use the zebrafish as a model organism, it is necessary to understand its physiology and behavioral characteristics well. Zebrafish belong to the class of teleosts, which constitutes $99 \%$ of the Actinopterygii species and emerged 350 million years ago. It inhabits vegetated freshwater streams and stagnant waters such as rice paddies in South and East Asia (Pakistan, Burma, India, and Nepal). Zebrafish live and lay eggs in shallow water at temperatures ranging from $24^{\circ} \mathrm{C}$ to $28^{\circ} \mathrm{C}$. Appropriate conductivity and $\mathrm{pH}$ values are between 10 and $271 \mu \mathrm{S}$ and $\mathrm{pH}$ 6.0-8.0, respectively (6). They feed on mosquito larvae and other insects. In the laboratory, embryos can hatch within 3 days after fertilization and mature within 2-3 months under good conditions (7). Zebrafish differ from previously used vertebrate models because of their external fertilization, transparency in development, small body 
size, short development time, production of a high number of eggs (200/female/week), and low maintenance costs (1).

Neurogenesis is defined as the progress of undifferentiated neural progenitor cells producing neurons that are mature and functional. The neural progenitor induction and the cell division phase are the first steps in neurogenesis that expands the progenitor pool. The specification of progenitors and differentiation into post-mitotic neurons follow the first step. All these steps are regulated to produce the various neuronal and glial cell types that will at the end form the mature central nervous system (CNS). Zebrafish have been used in many different studies on several concepts of neurogenesis. Unlike mammals, the external development of a zebrafish embryo makes zebrafish accessible for experimental manipulation in CNS research (8). Their small size and optical transparency allow observation of all processes in vivo throughout the early developmental stages, the early development of measurable behaviors, and the simplicity of the zebrafish nervous system compared to other model organisms provide advantages in nervous system studies $(9,10)$. Thanks to major advances in genetic, embryological, and optical techniques in recent years, zebrafish have become a unique vertebrate model for studying neurogenesis (8).

To date, most of the studies have focused on neurogenesis in the embryonic stages of zebrafish (8-10). However, recent research has reported that the brain of mature zebrafish can be a very useful model for the study of neurogenesis in the adult (1). Furthermore, in the early 1960s, new neurons were suggested to arise in the adult mammalian brain, in the hippocampus and olfactory bulb through labeling newly synthesized DNA by in-situ [3H]-thymidine (11). The production of new neurons is observed mainly in the subventricular zone and the subgranular zone of the telencephalon in rodents and primates (12). Unlike mammals, teleosts such as zebrafish present a higher potential of reproduction because nearly 16 regions of proliferation were found in different areas of adult zebrafish brains $(13,14)$. Approximately six thousand cells are produced in the adult zebrafish brain every 30 minutes which is the main reason for the popularity of zebrafish to study neurogenesis of the adult (15).

\section{Embryogenesis in Zebrafish}

In zebrafish, seven developmental stages are defined as the zygote, cleavage, blastula, gastrula, segmentation, pharyngeal, and hatching (exit from chorion) in the embryonic period (16). The time between the first cleavage (split) of the newly fertilized egg, approximately 40 minutes after fertilization, is called the zygote stage. The zygote is approximately $0.7-0.78 \mathrm{~mm}$ in diameter at fertilization. At the first cleavage 45 minutes after fertilization, two cells of equal size are formed. The cells or blastomeres then divide at intervals of about 15 minutes; 2 -cell structure $(0.75 \mathrm{~h}), 4$-cell structure $(1 \mathrm{~h}), 8$-cell structure $(1.25 \mathrm{~h})$, 16-cell structure (1.5 h), 32-cell structure (1.75 h), and the 64cell structure $(2 \mathrm{~h})$ is formed $(16,17)$.

Blastula refers to the period from the 128-cell structure, in which the blastodisc is ball-like, to the beginning of the gastrulation period. Structures formed during the blastula period are; 128 cell structure $(2.25 \mathrm{~h}), 256$-cell structure $(2.5 \mathrm{~h}), 512$-cell structure $(2.75 \mathrm{~h}), 1 \mathrm{k}$-cell structure, high stage $(3.3 \mathrm{~h})$, oblong phase (3.7 h), sphere phase ( $4 \mathrm{~h})$, dome phase ( $4.3 \mathrm{~h})$, and $30 \%$ epiboly phase structures $(4.7 \mathrm{~h})$. Stages of the gastrula stage include the formations of $50 \%$ epiboly ( $5.3 \mathrm{~h})$, germ ring ( $5.7 \mathrm{~h})$, shield stage (6 h), 75\% epiboly ( 8 h), $90 \%$ epiboly ( 9 h), and bud stage (10 h). The gastrula period is considered to be finished when the epiboly is complete and the tailbud is produced $(16,18)$.

The identification as an embryo until the end of the third day continues as the larval stage after hatching or not. The pectoral fin, gills, and jaw continue to develop rapidly. Monitoring the development of endodermal structures such as the digestive tract is difficult at this stage ( $48 \mathrm{~h}$ ) due to their deep location. The larval stage includes individuals that are no longer embryo but are not yet juvenile. The swim bladder is swollen, foraging and avoidance behaviors have started ( $72 \mathrm{~h}$ ). The juvenile stage is the stage in which sexual maturity is not achieved but most of the adult characteristics are acquired. In the laboratory, this period starts after about 4 weeks and lasts up to 6-12 weeks, depending on the breeding and rearing conditions (up to 89 days, according to www.zfin.org). The adult stage in fish is defined as the production of healthy gametes and secondary sexual characteristics that reach reproductive capability (16-18). Zebrafish's developmental timeline is summarized in Figure 1 (19).

\section{Zebrafish Developmental Timeline}

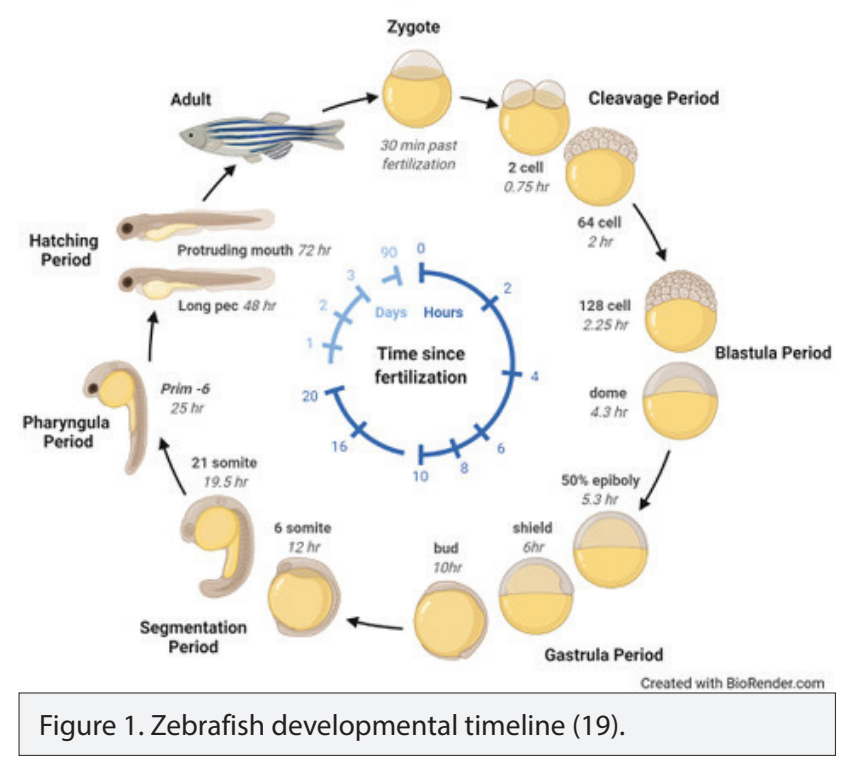

\section{Neurogenesis in Zebrafish Embryos}

Neuroectoderm is the first structure to be specified during the vertebrate nervous system development and this is named "neural induction" that occurs at the beginning of embryogenic development $(7,8)$. The mesodermal layer formed at the beginning of gastrulation folds in and interacts with the above ectoderm $(20,21)$. This possible layer of mesodermal secretes some local factors that activate or inhibit neural activation in 
the ectodermal layer. Cells in the ectodermal layer are thought to differentiate into neural tissue (22).

In recent years, vertebrate neural induction has been demonstrated by extrinsic signaling factors. The bone morphogenetic protein (BMP) is one of these factors to inhibit neural activity through epidermal regulation (23). Another factor is the wingless-integrated (Wnt) proteins that are among the earliest signals to regulate the formation of the neural plate primordium (24). The expression of Wnt8 has been shown in non-axial mesendoderm required for the recovery of hindbrain fate as well as the expression of $g b x 1$ in the hindbrain (25). The anterior neural plate is organized through the complex interaction of the Wnt signals and the Wnt antagonists, including TLC (26).

Signaling pathways, as well as molecules that regulate the substantial processes during embryogenesis, are substantial for developmental biology. The Wnt pathway is often related to different diseases, especially with endocrine diseases as well as cancer, showing that these diseases are due to some impaired developmental processes (27). In our previous studies, we have shown that different endocrine-disrupting chemicals such as bisphenol A and DEHP activated the Wnt/ $\beta$-catenin pathway in zebrafish embryos as evidenced by the increased expressions of c-myc which is the target gene and wnt3a $(28,29)$.

Another extrinsic factor is the fibroblast growth factor (Fgf). Fgf and the intrinsic transcription factors including the B1 (SoxB1) gene family are among the important cell proliferation modulators $(30,31)$. Significant progress has been made showing that it is based on complex interactions between these molecules. These secreted proteins allow neural fate to occur in the dorsal ectoderm and permit the formation of the neural plate. In particular, it is important to note that neural ectoderm is determined by members of the SoxB1 family (27). To date, the members of the SoxB1 family have been shown in zebrafish as sox 1 $(a / b)$, sox2, sox3, and sox19 (a/b) (28). Sox2 may be observed in the neural progenitors of zebrafish embryos and also in the neural stem cells of adult zebrafish brain $(23,32)$. Thus, Sox2 is a very important factor needed for the maintenance of neural progenitor traits and their related functions for the vertebrates (33). In summary, SoxB1 members together with the inhibitory role of BMP signaling and induction of Fgf signaling are substantial determinants to assert the neural stem cell pool during the zebrafish embryo early gastrulation stages (8).

In the neural plate, the initiation of neurogenesis is maintained through the proneural genes in the zebrafish which is observed during the late gastrulation phase. The neurog1 (neurogenin1) is one of the first proneural genes to produce transcription factors together with achaete-scute1 (asc1). These genes coding transcription factors are referred as the bHLH genes (33). On the other hand, shh has a key signal inducer role and is needed for the concentration-dependent activation of neurog 1 and ascl1, which causes the formation of thalamic nuclei in the vertebrates (34). In the midbrain-hindbrain boundary region of the neural tube of isthmus rhombocephali, regulation of the cellu- lar fate takes place via Fgf8 signaling (35). Through the initiation of neurogenesis, progenitor pool cells induce the formation of the differentiated cells within the midbrain-hindbrain boundary region (36). The activity of the progenitor pool is evident through her 5 and her 11 expression together with the inhibition of $\mathrm{E}(\mathrm{Spl})$ factors. These determinants inhibit the expression of the proneural genes including neurog1, ascl1a, as well as cyclin-dependent kinase inhibitors $(37,38)$.

The SoxB1 transcriptional targets are the $\mathrm{E}(\mathrm{Spl})$ family members which are the Her and Hes genes. They are named as the hairy genes, her 3,5,6,9 and 11. Ectopic Her and Hes gene expressions cause the downregulation of the expression of neurog 1 but loss-of-function trials reveal up-regulated neurog1 expression (38). SoxB1 together with the hairy genes leads to neurogenesis inhibition which is an important mechanism increasing the cell pool (39). Therefore, there is a critical mechanism that modulates the neurogenetic gradients during the embryonic central nervous system and this process is maintained spatially through the inhibition of neurogenesis, regulated through the local inhibition of the Her and Hes proteins $(8,40)$.

\section{Zebrafish Embryo as a Model Organism in Neurodevelop- mental Toxicity Studies}

Neurotoxicity is characterized as a detrimental effect on the structure and/or function of the nervous system and may result from exposure to food additives and environmental toxicants, as well as drugs used during chemotherapy, radiation therapy, and organ transplantation. Neurotoxicity is the second leading cause of drug withdrawals after cardiovascular toxicity. Diamthazole and vinyl chloride aerosol can be given as examples of some drug withdrawals due to neurotoxicity (41). Although many approved drugs have been shown to cause neurotoxic side effects, they do not have well-defined neurotoxicity profiles. For instance, an antibiotic, chloramphenicol and antituberculosis drugs such as ethambutol and isoniazid lead to cause optic neuritis (42). Antiepileptic drugs and chemotherapy agents, as well as aminoglycosides may lead to some cerebellar syndromes including dysarthria (43). Other drug-related neurological complications have also been reported including cognitive impairment, headache, and neuromuscular disorders (41-43).

Different approaches are defined to determine the neurotoxicity of a molecule and they are generally divided into three groups as the behavioral, the morphological or histopathological and finally the biochemical approach that evaluates the cellular metabolism and functions that have been changed. Currently, the preclinical analysis of neurotoxicity is generally based on the evaluation of behavioral abnormalities and/or the observation of clear histopathological lesions in the nervous tissue (44).

The most efficient methods to analyze neurotoxicity are the animal behavioral studies including aggression, survival, feeding, motor function, reproduction and, maternal behavior. These parameters may be adversely affected due to exposure to vari- 
ous neurotoxins. However, most effects on the central nervous system are mute and result in alterations in function, not easily identifiable in human studies, and are likely to cause minor changes in parameters including temperament, emotion, mood and, cognition that have not been sufficiently studied in animals (45). Histological methods are effective only when lesions in the nervous system are widespread and can be detected by immunohistochemical staining methods. Several specific biochemical markers (eg, alterations in the activity of enzymes and, protein phosphorylation) have been studied, but have only been shown to be beneficial for detecting specific types of neurotoxicity. There is a need to develop rapid assay methods and new animal models to predict neurotoxicity (46).

Zebrafish are very useful for neurotoxicity studies merging different approaches including genetic, cellular and, molecular methods. The main advantages of using zebrafish as a model organism in neurotoxicity research include its external development and genetic similarity to humans whereas, the main limitation may be suggested as its translational value (1). The main advantages and limitations of using zebrafish as a model organism in neurotoxicity research are summarized in Figure 2.

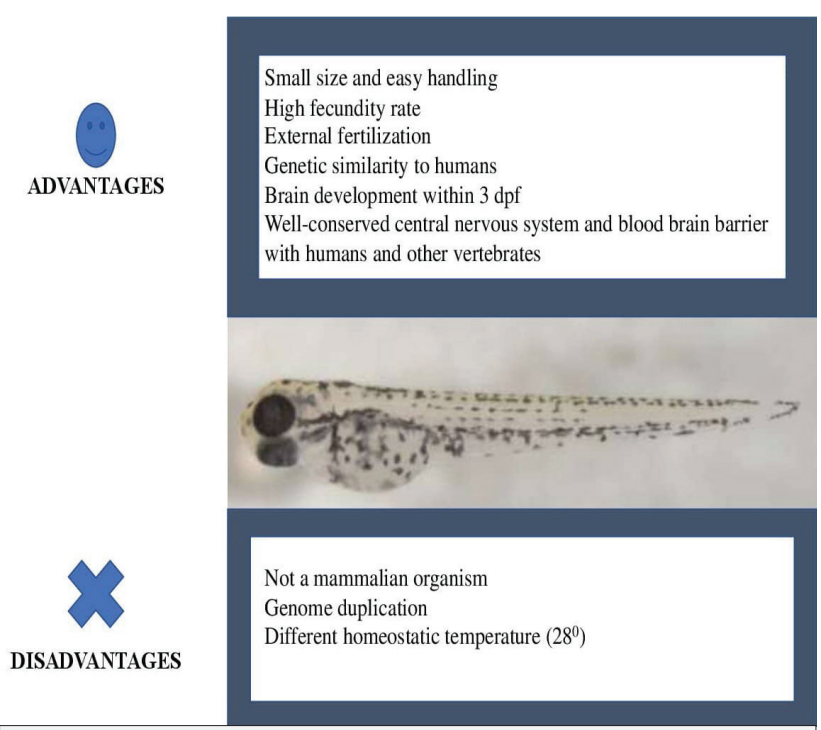

Figure 2. Advantages and disadvantages of using zebrafish as a model organism in neurotoxicity research.

Because the zebrafish embryo is transparent and develops rapidly, the formation of specific neurons and axon pathways can be screened in living embryos through differential interference contrast microscopy or by injecting live dyes (47). Special neuron types can be screened in whole mounted and fixed embryos by using immunohistochemistry or in situ hybridization (48). Motor neuron activity can be screened in vivo by using calcium imaging and patch-clamp recording (49).

The blood-brain barrier is a special type of capillary endothelial system that serves to protect the brain from substances that may be harmful in the bloodstream. It also provides the nutrients that are needed for the brain to regulate the main physio- logical functions. This barrier includes a complex system with special endothelial cells, macrophages, and astrocytes (50). The passage of macromolecules is prevented through tight junctions that are present between the endothelial cells (51). Lipid soluble, small molecules having molecular weight less than 400 daltons can pass the blood-brain barrier (50). The blood-brain barrier is also present in zebrafish and zebrafish is considered an excellent model organism to evaluate the molecular interactions as well as the permeability of drugs (52). It has been shown by Evans blue staining that blood-brain barrier functions are observed at $3 \mathrm{dpf}$ zebrafish and the transparent feature of zebrafish embryo provides a major advantage to study the penetration of drugs during development (53).

\section{Evaluation of Neurotoxicity in Zebrafish}

Zebrafish have a population of early neurons that are named as "primary neurons". These neurons are part of a comparably basic nervous system that differentiate to regulate the movement of the larva (54). The distribution and projection properties of specific primary neurons have been explained in literature thoroughly (55). At $24 \mathrm{~h}$ after fertilization ( $24 \mathrm{hpf}$ ), these primary neurons of zebrafish differentiate, and in this timeline, substantially large neuronal cells may be identified by using Normarski optics in vivo. Brain ventricles of zebrafish are formed at $48 \mathrm{hpf}$ (56). Zebrafish don't have a skull and, their body length may increase from $1 \mathrm{~mm}$ at $1 \mathrm{dpf}$ (day post fertilization) to $5 \mathrm{~mm}$ at $6 \mathrm{dpf}$. Whole animal staining can be done to comprehensively evaluate the whole nervous system. Classical neurotoxins investigated in zebrafish include dopaminergic neurotoxins including rotenone, 1-methyl-4-phenyl-1,2,3,6-tetrahydropyridine (MPTP), 6-hydroxydopamine (6-OHDA), and paraquat. non-NMDA-type glutamate receptor agonists or antagonists including domoic acid, 6-cyano-7-nitroquinoxaline-2,3-dione, alpha-latrotoxin, and picrotoxin; nicotinic acetylcholine receptor antagonists such as bungarotoxins and cobratoxins or acetylcholinesterase inhibitors; and the NMDA receptor antagonist, DL-2-amino-5-phosphonovalerate (AP-5) (1).

Neurotoxicity can be evaluated in zebrafish after exposure to a neurotoxic compound in the optic nerves, motor, and dopaminergic neurons as well as the myelin sheath. Moreover, the molecular mechanisms including oxidant/antioxidant pathways, apoptosis and proliferation assays can be observed directly in transparent fish. Accordingly, zebrafish have been shown to be sensitive to different neurotoxins such as ethanol, acrylamide, retinoic acid, neomycin, and tetrachlorodibenzo-p-dioxin $(57,58)$.

\section{Effects of Neurotoxins on Motor Neurons and Neuronal Pro- liferation}

In mammalian models, the effects of drug-induced neurotoxicity such as ethanol toxicity have been reported on motor neurons and also on neuronal proliferation. Ethanol has been reported to alter motor functions through the induction of motor neuron death and the inhibition of neuronal proliferation. Ethanol has also been found to affect brain and motor functions in humans (57). 
Similarly, the compounds that lead to neurotoxicity in humans have been reported to induce complementary neurotoxicity in zebrafish. Ethanol caused defects in the optic nerves as well as in the motor neurons and altered neuronal proliferation. 6-OHDA led to the oxidation of neurons and loss of dopaminergic neurons and L-2-hydroxyglutaric acid caused neuronal apoptosis (58).

\section{Effects of Neurotoxins on Dopaminergic Neurons}

In the midbrain, dopaminergic neurons are the major source of dopamine (DA) in the mammalian CNS, and the loss of DA is related to Parkinson's disease which is the most important human neurological disorder (1). Dopaminergic neurons are found in the substantia nigra pars compacta, which is rich in DA and contains neuromelanin and high levels of iron. Dopaminergic neurons have a substantial role in controlling many different brain functions such as, voluntary movement and a wide range of behavioral parameters including mood, reward, addiction, and stress. To evaluate the compound effects on dopaminergic neurons, 6-OHDA was administered as a neurotoxin that destroys catecholaminergic terminals, and then immunostained using an anti-tyrosine hydroxylase antibody. Results showed that 6-OHDA led to dopaminergic neurotoxicity $(1,59)$. Similarly, in mammals, 6-OHDA has been found to cause oxidative stress and result in neuronal death (59).

We have previously shown that as a neurotoxic pesticide that is capable of crossing the blood-brain barrier, rotenone exposure for 4 weeks led to decreased locomotor activity, dopamine, and serotonin levels and increased DOPAC and DOPAC/dopamine levels in zebrafish. Moreover, lipid peroxidation increased and antioxidant levels decreased suggesting the disrupted oxidant-antioxidant balance (60). We have also tested if exposure to rotenone disrupts the oxidant/antioxidant status in the intestine and brain of zebrafish and our results showed that lipid peroxidation increased whereas the activities of glutathione S-transferase and catalase decreased both in the intestinal tissues and brain of the rotenone exposed adult zebrafish (61).

Brain dopaminergic neurons of zebrafish embryos are sensitive to the MPTP. However, noradrenergic neurons in the medulla oblongata were not affected by MPTP exposure (62). Recently we have shown decreased locomotor activity in MPTP-exposed zebrafish embryos and morphine exerted neuroprotective effects against MPTP exposure by normalizing locomotor activity, acetylcholine esterase activity, Parkinson's Disease-related genes and oxidative stress (63). We have also shown the beneficial effects of 3-pyridineboronic in MPTP exposed zebrafish embryos by the amelioration of impaired locomotor activity and mitochondrial dysfunction (64). In zebrafish as the main dopamine-expressing neurons are located in the posterior tuberculum of the diencephalon decreased dopaminergic cells together with defects in swimming responses were also reported in zebrafish embryos (65).

\section{Effects of Neurotoxins on Mobility}

One of the biggest challenges in developing methods for assessing neurotoxicity is its association with behaviorally altered neuromorphological, neurochemical, and neurophysiological changes that are often considered abnormal movement. In zebrafish larvae, locomotor or motility patterns are stage-specific and behavioral abnormalities are easily discernible (66). Embryonic motor behavior develops sequentially; The early period consists of transient spontaneous curling contractions, followed by the appearance of twitching in response to touch, and later the ability to swim (67). At $4 \mathrm{dpf}$, the embryos are free-swimming and spontaneously change direction with characteristic ranges of speed and distance. Zebrafish also exhibit basic behaviors that include memory, non-associative learning, conditioned responses, and social behaviors such as schooling (68).

Various studies have shown that zebrafish mobility can be monitored using a computer-assisted motion detector (69). It has been shown that this assay format is useful for assessing compound-induced neurotoxicity (including seizures). Similar to results in mammals, pentylenetetrazole, a gamma-aminobutyric acid antagonist known to cause convulsions in humans, has been suggested to induce seizures in zebrafish. Moreover, electrophysiological, molecular changes, as well as the behavioral responses in zebrafish treated with pentylenetetrazole are similar to the effects reported in the rodent seizure model.

\section{Effects of Neurotoxins on Neurodevelopment}

Developmental neurotoxicity includes changes in behavior, neurohistology, neurochemistry, neurophysiology, or overall dysmorphology due to exposure during development. The developing embryonic brain is more susceptible to confusion caused by toxins due to the differentiation of specific cell types and the disruption of delicate processes that occur only during development, such as proliferation and migration of newly formed neurons (70). In addition, the developing brain is more exposed to blood-borne toxins before blood-brain barrier formation. Therefore, critical assessment of developmental neurotoxicity requires the use of an embryonic model.

In one study, well-characterized compounds were selected to validate zebrafish as a model of developmental neurotoxicity. Embryos were exposed by quasi-static immersion at stage $6 \mathrm{hpf}$ to 96 or $120 \mathrm{hpf}$; The induction of brain apoptosis or necrosis seen with the compounds was confirmed as an indicator of developmental neurotoxicity, and an effect on motor neurons in the caudal third of the embryo was shown to be associated with expected defects in motility. Different environmental pollutants were screened for their effects on brain apoptosis, axon tract and motor neuron formation, and catecholaminergic neurons in developing zebrafish, and compounds that act on several neurotoxicity parameters were identified. Some compounds even showed extensive toxicity at all neuroanatomical endpoints $(71,72)$. Overall, these results indicate a strong correlation with mammalian data, indicating that zebrafish is a suitable animal model for developmental neurotoxicity screening.

Harmful algal blooms (HABs) are formed when the growth of algae colonies derived from simple plants in sea and freshwater 
Table 1. Neurodevelopmental genes in zebrafish and their functions.

\begin{tabular}{lll}
\hline Gene name & Gene symbol & Function \\
\hline neurogenin 1 & neurog1 & Basal forebrain dopaminergic neuron determinant \\
\hline myelin basic protein a & $m b p$ & Structural constituent of myelin sheat \\
\hline a1-tubulin & $a 1$-tubulin & Brain development \\
\hline synapsin lla & syn2a & Synaptogenesis \\
\hline sonic hedgehog signaling molecule a & shha & Regulation of neurogenesis \\
\hline ELAV like neuron-specific RNA binding protein 3 & elavl3 & Regulation of neurogenesis \\
\hline
\end{tabular}

are out of control. HABs have toxic effects on people and animals. Recently HABs have been also shown to cause potential neurotoxins. For instance, domoic acid (DomA) is a potent HAB neurotoxin that can accumulate in shellfish and finfish under certain environmental conditions. Exposure to the DomA resulted in long-lasting behavioral deficits in rodents and primate models (73). In humans and nonhuman primates, oral exposure to DomA induced gastrointestinal effects, neurological symptoms, seizures, memory impairment, and limbic system degeneration. Rodents are less sensitive than humans or nonhuman primates and induce behavioral abnormalities. DomA exhibits similar neurotoxic effects across species from sea lions to zebrafish (74).

The drinking water of many different geographic areas including the US, Latin America, Asia, Africa is co-contaminated by neurotoxic pollutants such as inorganic arsenic and fluoride. Inorganic arsenic and fluoride affect the neurodevelopment of children due to the exposure during the post and pre-natal periods. Although, co-contamination with inorganic arsenic and fluoride can create more risk than their exposure alone, there is a lack of information about the concurent role in the deterioration of gut microbiota that acts as an organizer in neurodevelopment (75).

In zebrafish measuring the expression levels of different biomarkers is a sensitive and fast way to find out the alterations in the gene expression sequences in case of neurotoxin treatment. Different genes of the nervous system were suggested as potential neurotoxicity markers in zebrafish embryos that were treated with ethanol using RT-PCR. Neuronal stem cell transcripts were either decreased or increased during development, and the overexpression of an astrocytes marker was significant. It has been demonstrated that evaluation of the gene expression profile of the brain is useful in analyzing the neurotoxicity of many chemicals during development (76).

The microarray hybridization method has also been used to determine the effects of environmental toxins in zebrafish embryos and organ and cell-specific alterations in the gene expressions were detected by using in situ hybridization (77). When the genome profile and in situ studies were performed in methyl mercury exposed zebrafish embryos the gene expres- sions were found to be altered significantly in brain different neuronal subregions (78).

To evaluate the developmental effects of neurotoxins in zebrafish embryos gene expression levels of some specific markers are determined. For instance, myelin basic proteins (MBP) was determined in propofol (anesthetic) exposed zebrafish and decreased MBP expression was evident in the central nervous system (79).

Some other neurodevelopmental genes include syn $2 a$ and a1-tubulin that were found to be down-regulated in chlorpyrifos and 1,3-dichloro-2-propyl-phosphate exposed zebrafish embryos (80). On the other hand, neurog 1 is also an important neuro-developmental gene that is shown to be affected in ibuprofen and diclofenac exposed zebrafish embryos $(79,81)$. Ans also, elav/3, syn2a and, shha have been shown to be down regulated in triphenyl phosphate exposed zebrafish embryos (82). Zebrafish genes having specific roles in neurodevelopment are listed in Table 1.

Autism spectrum disorder (ASD) is a crucial disorder of neurodevelopment (83). Valproic acid, a pharmaceutical agent used to treat seizures, is suggested to be an ASD inducer, and defective social behavior has been shown in valproic acid exposed zebrafish embryos (84). The preliminary findings of our ongoing study show that valproic acid affects the expressions of shan$k 3 a, a d s /$ and $t s c 1 b$ that are genes related to ASD.

The utilization of gene profiling patterns can be suggested as a useful endpoint for neurotoxicity research to reveal the developmental neurotoxicity of some different and potentially toxic compounds.

\section{CONCLUSION}

Although zebrafish embryo and adult regulatory pathways are mainly different they share some similarities during the production of functional neurons. Embryonal neurons originate from the neuroectodermal epithelium whereas, adult neurons are derived from glia cells. Accordingly, it is important to reveal the specific differences between the embryo adult neurogenesis in terms of neurotoxicity research. Understanding the specific 
pathways of neurodevelopment will enable the improvement of therapeutic strategies. The translational value of zebrafish will also be improved through the incorporation of knowledge into the mammalian brain in terms of neuronal circuits.

Our review provides a brief overview of the emerging potential the zebrafish embryo has in neurotoxicity research during development. Although having some limitations, using the zebrafish as a model organism in neurotoxicology provides significant advantages. Many toxicity endpoints can be combined with different assays to evaluate the impact of large numbers of potential neurotoxic compounds.

We suggest the increasing employment of zebrafish in testing chemicals will speed up this process and facilitate the understanding of neurotoxicity mechanisms. Accordingly, we believe that the use of zebrafish and zebrafish embryos in research will become widespread, as in vivo imaging and analysis platforms, which are developing day by day, become more accessible.

Acknowledgement: This work was supported by the research grant from Marmara University Scientific Research Projects Commission (Grant number: TYL-2021-10205).

Informed Consent: Written consent was obtained from the participants.

\section{Peer Review: Externally peer-reviewed.}

Author Contributions: Conception/Design of Study- E.E.A.; Data Acquisition- S.C.; Data Analysis/Interpretation- S.C.; Drafting Manuscript- S.C.; Critical Revision of Manuscript- E.E.A.; Final Approval and Accountability- E.E.A., S.C.

Conflict of Interest: Authors declared no conflict of interest.

Financial Disclosure: Authors declared no financial support.

\section{REFERENCES}

1. Ünal I, Emekli-Alturfan E. Fishing for Parkinson's Disease: A review of the literature. J Clin Neurosci 2019; 62: 1-6.

2. Renier C, Faraco JH, Bourgin P, Motley T, Bonaventure P, Rosa F. Genomic and functional conservation of sedative-hypnotic targets in the zebrafish. Pharmacogenet Genomics 2007; 17: 237-53.

3. Rihel J, Prober DA, Arvanites A, Lam K, Zimmerman S, Jang S. Zebrafish behavioral profiling links drugs to biological targets and rest/wake regulation. Science 2010; 327: 348-51.

4. Howe K, Clark MD, Torroja CF, Torrance J, Berthelot C, Muffato M, et al. The zebrafish reference genome sequence and its relationship to the human genome. Nature 2013; 496: 498-503.

5. Kozol RA, Abrams AJ, James DM, Buglo E, Yan Q, Dallman JE. Function over form: modeling groups of inherited neurological conditions in zebrafish. Front Mol Neurosci 2016; 7: 9-55.

6. Engeszer RE, Patterson LB, Rao AA, Parichy DM. Zebrafish in the wild: A review of natural history and new notes from the field. Zebrafish 2007; 4: 21-40.

7. Bally-Cuif L, Vernier P. Organization and Physiology of the Zebrafish Nervous System. Fish Physiol 2010; 29: 25-80.

8. Schmidt R, Strähle U, Scholpp S. Neurogenesis in zebrafish - from embryo to adult. Neural Dev 2013; 8: 3.
9. Goulding M. Circuits controlling vertebrate locomotion: moving in a new direction. Nat Rev Neurosci 2009; 10: 507-18.

10. Brustein E, Saint-Amant L, Buss RR, Chong M, McDearmid JR, Drapeau P. Steps during the development of the zebrafish locomotor network. J Physiol Paris 2003; 97: 77-86.

11. Gould E, Gross CG. Neurogenesis in adult mammals: some progress and problems. J Neurosci 2002; 22(3): 619-23.

12. Gould E. How widespread is adult neurogenesis in mammals? Nat Rev Neurosci 2007; 8: 481-88.

13. Zupanc GKH, Hinsch K, Gage FH. Proliferation, migration, neuronal differentiation, and long-term survival of new cells in the adult zebrafish brain. J Comp Neurol 2005; 488: 290-319.

14. Grandel H, Kaslin J, Ganz J, Wenzel I, Brand M. Neural stem cells and neurogenesis in the adult zebrafish brain: origin, proliferation dynamics, migration and cell fate. Dev Biol 2006; 295: 263-77.

15. Hinsch K, Zupanc GKH. Generation and long-term persistence of new neurons in the adult zebrafish brain: a quantitative analysis. Neuroscience 2007; 146: 679-96.

16. Kimmel CB, Ballard WW, Kimmel SR, Ullmann B, Schilling TF. Stages of embryonic development of the zebrafish. Dev Dyn 1995: 203(3): 253-310.

17. Sasai $Y$, De Robertis EM. Ectodermal patterning in vertebrate embryos. Dev Biol 1997; 182: 5-20.

18. Spemann H, Mangold H. Induction of embryonic primordia by implantation of organizers from a different species. 1923. Int J Dev Biol 2001; 45: 13-38.

19. Zebrafish developmental timeline (Reprinted from "Zebrafish Developmental Timeline", by BioRender.com (2021). Retrieved from https://app.biorender.com/biorender-templates)

20. Doniach T, Musci TJ. Induction of anteroposterior neural pattern in Xenopus: evidence for a quantitative mechanism. Mech Dev 1995; 53: 403-13.

21. Lumsden A, Krumlauf R. Patterning the vertebrate neuraxis. Science 1996; 274: 1109-15.

22. Weinstein DC, Hemmati-Brivanlou A. Neural induction. Annu Rev Cell Dev Biol 1999; 15: 411-33.

23. Okuda Y, Ogura E, Kondoh H, Kamachi Y. B1 SOX coordinate cell specification with patterning and morphogenesis in the early zebrafish embryo. PloS Genet 2010; 6(5): e1000936.

24. Kim $\mathrm{CH}$, Oda $\mathrm{T}$, Itoh $\mathrm{M}$, Jiang $\mathrm{D}$, Artinger KB, Chandrasekharappa SC, et al. Repressor activity of Headless/Tcf3 is essential for vertebrate head formation. Nature 2000; 407: 913-16.

25. Rhinn M, Lun K, Luz M, Werner M, Brand M. Positioning of the midbrain-hindbrain boundary organizer through global posteriorization of the neuroectoderm mediated by Wnt8 signaling. Development 2005; 132: 1261-72.

26. Houart C, Westerfield M, Wilson SW. A small population of anterior cells patterns the forebrain during zebrafish gastrulation. Natur 1998; 391: 788-92.

27. Üstündağ ÜV, Emekli-Alturfan E. Wnt pathway: A mechanism worth considering in endocrine disrupting chemical action. Toxicol Ind Health 2020; 36(1): 41-53.

28. Üstündağ ÜV, Ünal İ, Ateş PS, Alturfan AA, Yiğitbaşı T, Emekli-Alturfan E. Bisphenol A and di(2-ethylhexyl) phthalate exert divergent effects on apoptosis and the $\mathrm{Wnt} / \mathrm{\beta}$-catenin pathway in zebrafish embryos: A possible mechanism of endocrine disrupting chemical action. Toxicol Ind Health 2017; 33(12): 901-10.

29. Bielen $\mathrm{H}$, Houart C. BMP signaling protects telencephalic fate by repressing eye identity and its $\mathrm{Cxcr4-dependent} \mathrm{morphogenesis.}$ Dev Cell 2012; 23: 812-22.

30. Stern CD. Neural induction: 10 years on since the "default model" Curr Opin Cell Biol 2006; 18: 692-7. 
31. Penzel R, Oschwald R, Chen Y, Tacke L, Grunz H. Characterization and early embryonic expression of a neural specific transcription factor xSOX3 in Xenopus laevis. Int J Dev Biol 1997; 41: 667-77.

32. Dee CT, Hirst CS, Shih Y-H, Tripathi VB, Patient RK, Scotting PJ. Sox3 regulates both neural fate and differentiation in the zebrafish ectoderm. Dev Biol 2008; 320: 289301.

33. Kaslin J, Ganz J, Geffarth M, Grandel H, Hans S, Brand M. Stem cells in the adult zebrafish cerebellum: initiation and maintenance of a novel stem cell niche. J Neurosci 2009; 29: 6142-53.

34. Bani-Yaghoub M, Tremblay RG, Lei JX, Zhang D, Zurakowski B, Sandhu JK, et al. Role of Sox2 in the development of the mouse neocortex. Dev Biol 2006; 295: 52-66.

35. Allende ML, Weinberg ES. The expression pattern of two zebrafish achaete-scute homolog (ash) genes is altered in the embryonic brain of the cyclops mutant. Dev Biol 1994; 166: 509-30.

36. Scholpp S, Delogu A, Gilthorpe J, Peukert D, Schindler S, Lumsden A. Her6 regulates the neurogenetic gradient and neuronal identity in the thalamus. Proc Natl Acad Sci U S A 2009; 106: 19895-900.

37. Tallafuss A, Adolf B, Bally-Cuif L. Selective control of neuronal cluster size at the forebrain/midbrain boundary by signaling from the prechordal plate. Dev Dyn 2003; 227: 524-35.

38. Geling A, Itoh M, Tallafuss A, Chapouton $P$, Tannhäuser B, Kuwada JY, Chitnis AB, Bally-Cuif L. bHLH transcription factor Her5 links patterning to regional inhibition of neurogenesis at the midbrain-hindbrain boundary. Development 2003; 130: 1591-604.

39. Ninkovic J, Tallafuss A, Leucht C, Topczewski J, Tannhäuser B, Solnica-Krezel L, Bally-Cuif L. Inhibition of neurogenesis at the zebrafish midbrain-hindbrain boundary by the combined and dose-dependent activity of a new hairy/E(spl) gene pair. Development 2005; 132(1): 75-88.

40. Lyons DA, Guy AT, Clarke JDW. Monitoring neural progenitor fate through multiple rounds of division in an intact vertebrate brain. Development 2003; 130: 3427-36.

41. Wysowski DK, Swartz L. Adverse drug event surveillance and drug withdrawals in the United States, 1969-2002: the importance of reporting suspected reactions. Arch Intern Med 2005; 165(12): 13639.

42. Kulkarni HS, Keskar VS, Bavdekar SB, Gabhale Y. Bilateral optic neuritis due to isoniazid (INH). Indian Pediatr 2010; 47(6): 533-5.

43. Edson RS, Terrell CL. The aminoglycosides. Mayo Clin Proc 1999; 74(5): 519-28.

44. Moser VC. Functional assays for neurotoxicity testing. Toxicol Pathol 2011; 39(1): 36-45.

45. Kulig B, Alleva E, Bignami G, Cohn J, Cory-Slechta D, Landa V, et al. Animal behavioral methods in neurotoxicity assessment: SGOMSEC joint report. Environ Health Perspect 1996; 104(Suppl 2): 193204.

46. Kung MP, Kostyniak PJ, Olson JR, Sansone FM, Nickerson PA, Malone MA, et al. Cell specific enzyme markers as indicators of neurotoxicity: effects of acute exposure to methylmercury. Neurotoxicology 1989; 10(1): 41-52.

47. Kuwada JY, Bernhardt RR, Nguyen N. Development of spinal neurons and tracts in the zebrafish embryo. J Comp Neurol 1990; 302(3): 617-28.

48. Moens $C B$, Fritz A. Techniques in neural development. Methods Cell Biol 1999; 59: 253-72.

49. Brustein E, Saint-Amant L, Buss RR, Chong M, McDearmid JR, Drapeau P. Steps during the development of the zebrafish locomotor network. J Physiol Paris 2003; 97(1): 77-86.

50. Ballabh P, Braun A, Nedergaard M. The blood-brain barrier: an overview: structure, regulation, and clinical implications. Neurobiol Dis 2004; 16(1): 1-13.
51. Engelhardt B. Development of the blood-brain barrier. Cell Tissue Res 2003; 314(1): 119-29.

52. Cserr HF, Bundgaard M. Blood-brain interfaces in vertebrates: a comparative approach. Am J Physiol 1984; 246(3): R277-88.

53. Eliceiri BP, Gonzalez AM, Baird A. Zebrafish model of the bloodbrain barrier: morphological and permeability studies. Methods Mol Biol 2011; 686: 371-8.

54. Kimmel CB, Miller CT, Kruze G, Ullmann B, BreMiller RA, Larison KD, Snyder HC. The shaping of pharyngeal cartilages during early development of the zebrafish. Dev Biol 1998; 203(2): 245-63.

55. Eisen JS, Pike SH. The spt-1 mutation alters segmental arrangement and axonal development of identified neurons in the spinal cord of the embryonic zebrafish. Neuron 1991; 6(5): 767-76.

56. Schier AF, Neuhauss SC, Harvey M, Malicki J, Solnica-Krezel L, Stainier DY, et al. Mutations affecting the development of the embryonic zebrafish brain. Development 1996; 123: 165-78.

57. Biller A, Bartsch AJ, Homola G, Solymosi L, Bendszus M. The effect of ethanol on human brain metabolites longitudinally characterized by proton MR spectroscopy. J Cereb Blood Flow Metab 2009; 29(5): 891-902.

58. Parng C, Roy NM, Ton C, Lin Y, McGrath P. Neurotoxicity assessment using zebrafish. J Pharmacol Toxicol Methods 2007; 55(1): 103-12.

59. Elkon H, Melamed E, Offen D. Oxidative stress, induced by 6-hydroxydopamine, reduces proteasome activities in PC12 cells. J Mol Neurosci 2004; 24: 387-400.

60. Ünal İ, Çalışkan-Ak E, Üstündağ ÜV, Ateş PS, Alturfan AA, Altinoz $M A$,et al. Neuroprotective effects of mitoquinone and oleandrin on Parkinson's disease model in zebrafish. Int J Neurosci 2020; 130(6): 574-82.

61. Ünal İ, Üstündağ ÜV, Ateş PS, Eğilmezer G, Alturfan AA, Yiğitbaşı T, et al. Rotenone impairs oxidant/antioxidant balance both in brain and intestines in zebrafish. Int J Neurosci 2019; 129(4): 363-68.

62. McKinley ET, Baranowski TC, Blavo DO, Cato C, Doan TN, Rubinstein AL. Neuroprotection of MPTP-induced toxicity in zebrafish dopaminergic neurons. Brain Research. Molecular Brain Research 2005; 141(2): 128-37.

63. Cansiz D, Ustundag UV, Unal I, Alturfan AA, Emekli-Alturfan E. Morphine attenuates neurotoxic effects of MPTP in zebrafish embryos by regulating oxidant/antioxidant balance and acetylcholinesterase activity. Drug Chem Toxicol 2021; 2: 1-9.

64. Üstündağ FD, Ünal İ, Cansız D, Üstündağ ÜV, Subaşat HK, Alturfan $A A$, et al. 3-Pyridinylboronic acid normalizes the effects of 1-Methyl-4-phenyl-1,2,3,6-tetrahydropyridine exposure in zebrafish embryos. Drug Chem Toxicol 2020; 21: 1-8.

65. Lam CS, Korzh V, Strahle U. Zebrafish embryos are susceptible to the dopaminergic neurotoxin MPTP. The European Journal of Neuroscience 2005; 21(6): 1758-62.

66. Granato $M$, Nüsslein-Volhard C. Fishing for genes controlling development. Curr Opin Genet Dev 1996; 6(4): 461-8.

67. Drapeau P, Saint-Amant L, Buss RR, Chong M, McDearmid JR, Brustein E. Development of the locomotor network in zebrafish. Prog Neurobiol 2002; 68(2): 85-111.

68. Best JD, Alderton WK. Zebrafish: An in vivo model for the study of neurological diseases. Neuropsychiatr Dis Treat 2008; 4(3): 567-76.

69. Winter MJ, Redfern WS, Hayfield AJ, Owen SF, Valentin JP, Hutchinson TH. Validation of a larval zebrafish locomotor assay for assessing the seizure liability of early-stage development drugs. J Pharmacol Toxicol Methods 2008; 57(3): 176-87.

70. Rice D, Barone S Jr. Critical periods of vulnerability for the developing nervous system: evidence from humans and animal models. Environ Health Perspect 2000; 108(3): 511-33. 
71. Ton C, Lin Y, Willett C. Zebrafish as a model for developmental neurotoxicity testing. Birth Defects Res A Clin Mol Teratol 2006; 76: 553-67.

72. Legradi JB, Di Paolo C, Kraak MHS, van der Geest HG, Schymanski EL, Williams AJ, et al. An ecotoxicological view on neurotoxicity assessment. Environ Sci Eur 2018; 30(1): 46.

73. Panlilio JM, Aluru N, Hahn ME. Developmental Neurotoxicity of the Harmful Algal Bloom Toxin Domoic Acid: Cellular and Molecular Mechanisms Underlying Altered Behavior in the Zebrafish Model. Environ Health Perspect. 2020; 128(11): 117002.

74. Costa LG, Giordano G, Aschner M. Domoic Acid. Aminoff MJ, Daroff RB, editors. Encyclopedia of the Neurological Sciences. 2nd ed. Massachusetts: Academic Press; 2014. p. 1016-17.

75. Qiu Y, Chen X, Yan X, Wang J, Yu G, Ma W, et al. Gut microbiota perturbations and neurodevelopmental impacts in offspring rats concurrently exposure to inorganic arsenic and fluoride. Environ Int. 2020; 140: 105763.

76. Fan CY, Cowden J, Simmons SO, Padilla S, Ramabhadran R. Gene expression changes in developing zebrafish as potential markers for rapid developmental neurotoxicity screening. Neurotoxicol. Teratol 2010; 32: 91-8.

77. Yang L, Kemadjou JR, Zinsmeister C, Bauer M, Legradi J, Müller F, et al. Transcriptional profiling reveals barcode-like toxicogenomic responses in the zebrafish embryo. Genome Biol 2007; 8(10): R227.
78. Ho NY, Yang L, Legradi J, Armant O, Takamiya M, Rastegar S, et al. Gene responses in the central nervous system of zebrafish embryos exposed to the neurotoxicant methyl mercury. Environ. Sci. Technol 2013; 47: 3316-3325.

79. Xia L, Zheng L, Zhou JL. Effects of ibuprofen, diclofenac and paracetamol on hatch and motor behavior in developing zebrafish (Danio rerio). Chemosphere 2017; 182: 416-25.

80. Jeong JY, Einhorn Z, Mercurio S, Lee S, Lau B, Mione M, Wilson SW, Guo S. Neurogenin 1 is a determinant of zebrafish basal forebrain dopaminergic neurons and is regulated by the conserved zinc finger protein Tof/Fezl. Proc Natl Acad Sci U S A. 2006; 28; 103(13): 5143-8.

81. Li R, Zhang L, Shi Q, Guo Y, Zhang W, Zhou B. A protective role of autophagy in TDCIPP-induced developmental neurotoxicity in zebrafish larvae. Aquat Toxicol 2018; 199: 46-54.

82. Shi Q, Wang M, Shi F, Yang L, Guo Y, Feng C, et al. Developmental neurotoxicity of triphenyl phosphate in zebrafish larvae. Aquat Toxicol 2018; 203: 80-7.

83. Lee S, Chun HS, Lee J, Park HJ, Kim KT, Kim CH, et al. Plausibility of the zebrafish embryos/larvae as an alternative animal model for autism: A comparison study of transcriptome changes. PloS one 2018; 13(9): e0203543.

84. Chen J, Lei L, Tian L, et al. Developmental and behavioral alterations in zebrafish embryonically exposed to valproic acid (VPA): An aquatic model for autism. Neurotoxicol Teratol 2018; 66: 8-16. 